\title{
MIXED GERM TUMOR WITH YOLK SAC TUMOR AND MATURE TERATOMA - CASE REPORT AND LITERATURE REVIEW
}

\section{K.A.S. Almeida1, G. Carvalho², J.L. Bravin¹, M.I.P.D.S. Vianna³, V.H.D.S. Vicuna1, I.C.}

Menezes ${ }^{1}$, V.D. Santana ${ }^{1}$.

${ }^{1}$ Instituto Nacional do Cancer José de Alencar, INCA, Oncological Surgery - Resident, Rio de Janeiro, Brazil.

${ }^{2}$ Instituto Nacional do Cancer José de Alencar, INCA, Oncological Surgery, Rio de Janeiro, Brazil.

IInstituto Nacional do Cancer José de Alencar, INCA, Pathologist, Rio de Janeiro, Brazil.

Objectives: This study aims to report a case of mixed germ cell tumor in patients treated at the National Cancer Institute (INCA) in the city of Rio de Janeiro - Brazil Methods: Physical record review and literature review

Results: Germ cell tumors are rare and have highly malignant subtypes, such as the endodermal sinus tumor. In the present case: a female patient, 18 years old, referred to INCA after being submitted to surgical excision in another hospital, an initial diagnosis of stage IV ovary embryonal carcinoma. After evaluation, chemotherapy (BEP - bleomycin, etoposide and cisplatin) was chosen. In spite of the systemic treatment, the lesions progressed leading to patient clinical worsening (decrease of the general state and increase in ascites). Discussed case in clinical meeting and deliberate surgical rescue. 26/02/2019: Pelvic mass resection with approximately $30 \mathrm{~kg}$, right annexectomy and resection of satellite lesions in the pelvis, peri-splenic, omentum and hepatic nodulectomy of 3.5 $\mathrm{cm}$ in segment VII. The freezing biopsy found mucinous neoplasia related to teratoma. The definitive diagnosis was of mixed germ tumor with endodermal sinus tumor and mature teratoma.

Conclusions: The standard treatment for advanced stages for this neoplasm is surgery followed by adjuvant chemotherapy, but in relapses treatment is still controversial. This case reflects the difficult decision between clinical and surgical treatment in rare and selected

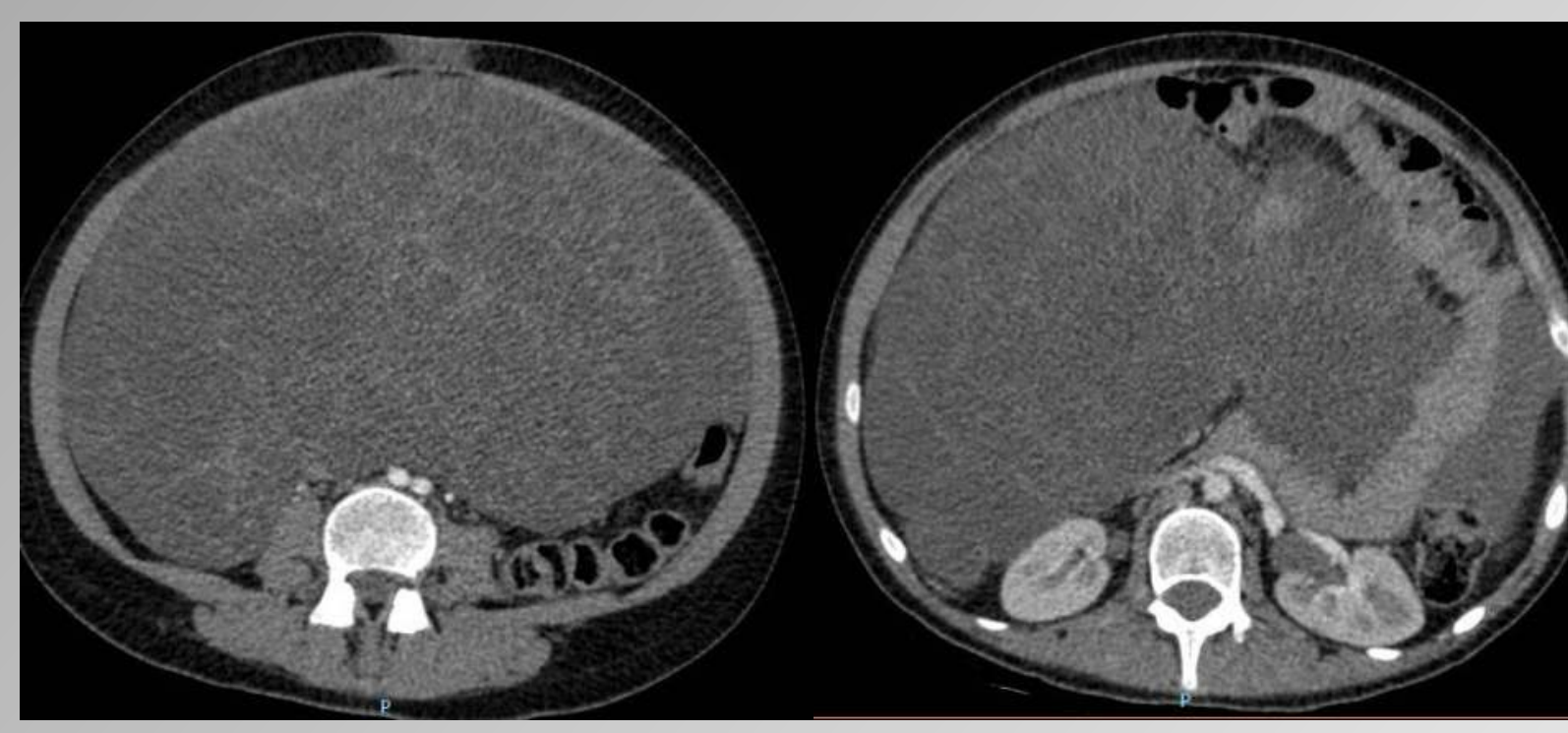

Figure 1. CT scan showing extensive pelvic mass

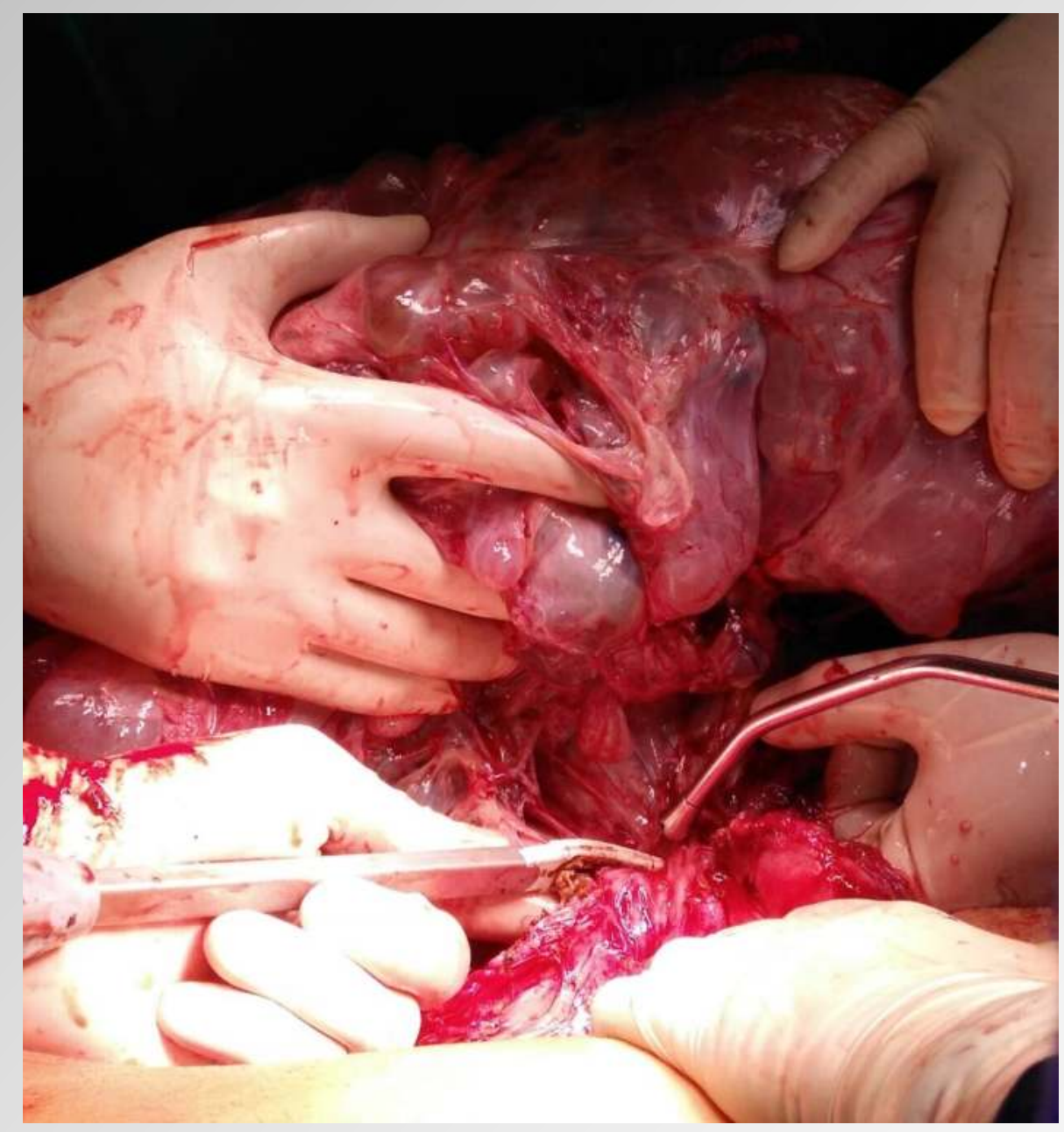

Figure 2. Tumor pedicle dissection

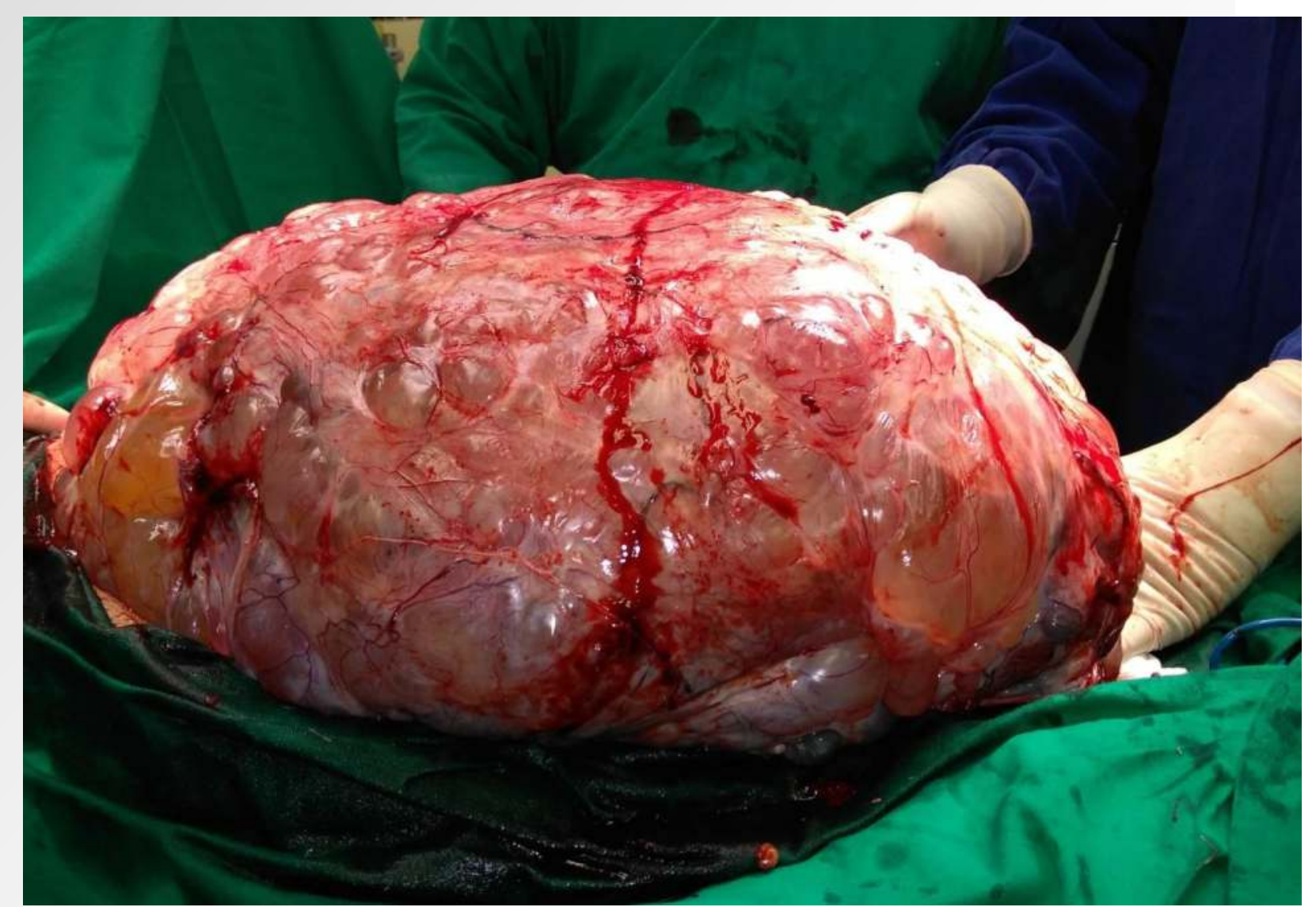

Figure 3. Fully rescted pelvic mass of approximately $30 \mathrm{~kg}$. cases. 